\title{
Positive Solutions of a Singular Third-Order $m$-Point Boundary Value Problem
}

\author{
Shaolin Zhou and Xiaoling Han \\ College of Mathematics and Information Science, Northwest Normal University, Lanzhou 730070, China \\ Correspondence should be addressed to Xiaoling Han; hanxiaoling@nwnu.edu.cn
}

Received 4 December 2012; Accepted 16 January 2013

Academic Editor: To Ma

Copyright (C) 2013 S. Zhou and X. Han. This is an open access article distributed under the Creative Commons Attribution License, which permits unrestricted use, distribution, and reproduction in any medium, provided the original work is properly cited.

This paper is concerned with the existence and nonexistence of positive solutions to the singular third-order $m$-point boundary value problem $u^{\prime \prime \prime}(t)+a(t) f(u(t))=0,0<t<1, u(0)=u^{\prime}(0)=0, u^{\prime}(1)-\sum_{i=1}^{m-2} \alpha_{i} u^{\prime}\left(\xi_{i}\right)=\lambda$, where $\xi_{i} \in[0,1), \alpha_{i} \in[0, \infty)(i=$ $1,2, \ldots, m-2)$ are constants, $\lambda \in(0,1)$ is a parameter, $f:[0, \infty) \rightarrow[0, \infty)$ is continuous and $a(\cdot)$ is allowed to be singular at $t=0$ and $t=1$. The results here essentially extend and improve some known results.

\section{Introduction and the Main Results}

Singular boundary value problems for nonlinear ordinary differential equations arise in a variety of areas of applied mathematics, physics, chemistry, and so on. For earlier works, see [1-3]. Nonsingular third-order multipoint boundary value problems have been studied by many authors by using different type of techniques, see, for example, [4-9] and the references therein. In recent years, singular third-order multipoint boundary value problems have also received much attention, see [10-12].

Very recently, motivated by Ma [13], Sun [14] considered the third-order three-point boundary value problem

$$
\begin{aligned}
& u^{\prime \prime \prime}(t)+a(t) f(u(t)) 0=0, \quad t \in(0,1), \\
& u(0)=u^{\prime}(0)=0, \quad u^{\prime}(1)-\alpha u^{\prime}(\eta)=\lambda,
\end{aligned}
$$

where $\eta \in(0,1), \alpha \in[0,1 / \eta)$ are constants and $\lambda \in(0, \infty)$ is a parameter. Under the following assumptions:

$\left(H_{1}\right) a \in C((0,1),[0, \infty))$ and $0<\int_{0}^{1}(1-s) s a(s) d s<\infty$;

$\left(H_{2}\right) f \in C([0, \infty),[0, \infty))$;

$\left(H_{4}^{*}\right) f$ is superlinear, that is, $f_{0}=0, f_{\infty}=\infty$;

$\left(H_{5}^{*}\right) f$ is sublinear, that is, $f_{0}=\infty, f_{\infty}=0$, where $f_{0}=\lim _{r \rightarrow 0^{+}}(f(r) / r), f_{\infty}=\lim _{r \rightarrow+\infty}(f(r) / r)$.

By using Guo-Krasnosel'skii fixed point theorem, the author established the following results.
Theorem A (see [14, Theorem 3.1]). Suppose that $\left(H_{1}\right),\left(H_{2}\right)$, and $\left(H_{4}^{*}\right)$ hold. Then the problem $\left(P_{\lambda}\right)$ has at least one positive solution for $\lambda$ small enough and has no positive solution for $\lambda$ large enough.

Theorem B (see [14, Theorem 3.2]). Suppose that $\left(H_{1}\right),\left(H_{2}\right)$, and $\left(H_{4}^{*}\right)$ hold. If $f$ nondecreasing, then there exists a positive constant $\lambda^{*}$ such that the problem $\left(P_{\lambda}\right)$ has at least one positive solution for $\lambda \in\left(0, \lambda^{*}\right)$ and has no positive solution for $\lambda \epsilon$ $\left(\lambda^{*}, \infty\right)$.

Theorem C (see [14, Theorem 3.3]). Suppose that $\left(H_{1}\right),\left(H_{2}\right)$, and $\left(H_{5}^{*}\right)$ hold. Then the problem $\left(P_{\lambda}\right)$ has at least one positive solution for any $\lambda \in(0, \infty)$.

Being directly inspired by the previously mentioned works, we will consider the existence and nonexistence of positive solutions to the following third-order $m$-point BVP:

$$
\begin{gathered}
u^{\prime \prime \prime}(t)+a(t) f(u(t))=0, \quad 0<t<1, \\
u(0)=u^{\prime}(0)=0, \quad u^{\prime}(1)-\sum_{i=1}^{m-2} \alpha_{i} u^{\prime}\left(\xi_{i}\right)=\lambda,
\end{gathered}
$$

where $\xi_{i} \in[0,1), \alpha_{i} \in[0, \infty)(i=1,2, \ldots, m-2)$ are constants and $\lambda \in(0,1)$ is a parameter, $a(\cdot)$ is allowed to be singular at $t=0$ and $t=1$. Here, the solution $u$ of BVP of (1), 
$\left(2_{\lambda}\right)$ is called positive solution if $u(t)$ is positive on $(0,1)$ and satisfies (1) and the boundary conditions $\left(2_{\lambda}\right)$.

We assume that $\left(H_{1}\right),\left(H_{2}\right)$ hold and make the following additional assumptions:

$$
\left(H_{3}\right) 0<\sum_{i=1}^{m-2} \alpha_{i} \xi_{i}<1
$$

$\left(H_{4}\right) \lim _{u \rightarrow 0^{+}} \sup (f(u) / u)<\Lambda_{1}$ and $\lim _{u \rightarrow+\infty} \inf (f(u) /$ u) $>\Lambda_{2}$;

$\left(H_{5}\right) \lim _{u \rightarrow 0^{+}} \inf (f(u) / u)>0$ and $\lim _{u \rightarrow+\infty} \sup (f(u) /$ $u)<L$,

where

$$
\begin{gathered}
\Lambda_{1}=2(1+\|h\|)^{-1} L, \quad\|h\|=\frac{1}{2} \gamma, \\
L=\left[\left(1+\gamma \sum_{i=1}^{m-2} \alpha_{i}\right) \int_{0}^{1}(1-s) s a(s) d s\right]^{-1}, \\
\gamma=\left(1-\sum_{i=1}^{m-2} \alpha_{i} \xi_{i}\right)^{-1}, \\
\Lambda_{2}=\frac{1}{4}\left[\theta^{2} \int_{\theta}^{1}(1-s) s a(s) d s\right]^{-1}
\end{gathered}
$$

From $\left(H_{1}\right)$, we know that there exists $t_{0} \in(0,1)$ such that $a\left(t_{0}\right)>0$. Let $\theta$ satisfy $0<\theta<t_{0}<1$.

Our main results are the following.

Theorem 1. Let $\left(H_{1}\right)-\left(H_{4}\right)$ hold. Then there exists a positive number $\lambda^{*}$ such that BVP of $(1),\left(2_{\lambda}\right)$ has at least one positive solution for $\lambda \in\left(0, \lambda^{*}\right)$ and none for $\lambda \in\left(\lambda^{*}, \infty\right)$.

Theorem 2. Let $\left(\mathrm{H}_{1}\right)-\left(\mathrm{H}_{3}\right)$ and $\left(\mathrm{H}_{5}\right)$ hold. Then BVP of (1), $\left(2_{\lambda}\right)$ has at least one positive solution for any $\lambda \in(0, \infty)$.

The proof of previous theorems is based on the Schauder fixed-point theorem.

Remark 3. BVP $\left(P_{\lambda}\right)$ is a special case of $(1),\left(2_{\lambda}\right)$ with $\alpha_{1}=$ $\alpha, \xi_{1}=\eta$, and $\alpha_{2}=\cdots=\alpha_{m-2}=0, \xi_{2}=\cdots=\xi_{m-2}=0$.

Remark 4. $\left(H_{4}\right)$ allows but do not require the nonlinearity $f(u)$ to be sublinear at zero and infinity; $\left(H_{5}\right)$ allows but do not require the nonlinearity $f(u)$ to be sublinear at zero and infinity.

Remark 5. We do not assume any monotonicity condition on the nonlinearity as in [14]. We find that the nondecreasing condition of $f$ can be removed from Theorem 3.2 in [14], and the same result is obtained in Theorem 1 .

Remark 6. It is obvious that Theorem 1 is an extension and complement of Theorems 3.1 and 3.2; furthermore, Theorem 2 is also an extension of Theorem 3.3 in [14].

\section{Preliminary Lemmas}

In this section, we present some notation and preliminary lemmas.
Let $C^{+}[0,1]=\{u \in C[0,1] \mid u(t) \geq 0, t \in[0,1]\}$ equipped with the norm $\|u\|=\max _{0 \leq t \leq 1}|u(t)|$.

Lemma 7 (see [15, Lemma 2.1]). Suppose that $\phi \in\{\varphi \in$ $\left.L_{\text {loc }}^{1}[0,1]\left|\int_{0}^{1} t(1-t)\right| \varphi(t) \mid d t<\infty\right\}$.

(i) Then $\int_{0}^{t} s \phi(s) d s, \int_{t}^{1}(1-s) \phi(s) d s \in L^{1}(0,1)$ and

$$
\begin{aligned}
\int_{0}^{1} \int_{0}^{t} s \phi(s) d s d t & =\int_{0}^{1} \int_{t}^{1}(1-s) \phi(s) d s d t \\
& =\int_{0}^{1} s(1-s) \phi(s) d s .
\end{aligned}
$$

(ii) Let $r \in(0,1)$. Then

$$
\lim _{t \rightarrow 0^{+}} v(t) \int_{t}^{1}(1-s) \phi(s) d s=0,
$$

for every $v \in C^{1}[0, r]$ with $v(0)=0$, and

$$
\lim _{t \rightarrow 1^{-}} w(t) \int_{0}^{t} s \phi(s) d s=0,
$$

for every $w \in C^{1}[r, 1]$ with $w(1)=0$.

Lemma 8. Suppose that $\left(H_{1}\right)-\left(H_{3}\right)$ hold, then BVP

$$
\begin{gathered}
u^{\prime \prime \prime}(t)+a(t) f(u(t))=0, \quad 0<t<1, \\
u(0)=u^{\prime}(0)=0, \quad u^{\prime}(1)-\sum_{i=1}^{m-2} \alpha_{i} u^{\prime}\left(\xi_{i}\right)=0,
\end{gathered}
$$

has a unique nonnegative solution $u \in C^{1}[0,1] \cap C^{3}(0,1)$ which can be represented as

$$
u(t)=\int_{0}^{1} G(t, s) a(s) f(u(s)) d s+\frac{1}{2} \gamma \delta_{f} t^{2},
$$

where

$$
\begin{gathered}
G(t, s):=\frac{1}{2} \begin{cases}\left(2 t-t^{2}-s\right) s, & 0 \leq s \leq t \leq 1, \\
(1-s) t^{2}, & 0 \leq t \leq s \leq 1,\end{cases} \\
G_{1}(t, s):= \begin{cases}(1-t) s, & 0 \leq s \leq t \leq 1, \\
(1-s) t, & 0 \leq t \leq s \leq 1,\end{cases}
\end{gathered}
$$

and $\delta_{f}=\sum_{i=1}^{m-2} \alpha_{i} \int_{0}^{1} G_{1}\left(\xi_{i}, s\right) a(s) f(u(s)) d s$.

Proof. The proof of the uniqueness is standard and hence is omitted here. Now we prove the existence of the solution.

From $\left(H_{1}\right)-\left(H_{3}\right)$ and Lemma 7 , we conclude that the integration in (7) is well defined. Let $v=u^{\prime}$; then BVP (6) may be reduced to boundary value problems

$$
\begin{gathered}
v^{\prime \prime}(t)+a(t) f(u(t))=0, \quad 0<t<1, \\
v(0)=0, \quad v(1)-\sum_{i=1}^{m-2} \alpha_{i} v\left(\xi_{i}\right)=0, \\
u^{\prime}(t)=v(t), \quad 0<t<1, \\
u(0)=0 .
\end{gathered}
$$


We claim that (9), (10) have a nonnegative solution $v$ which can be represented as

$$
v(t)=\int_{0}^{1} G_{1}(t, s) a(s) f(u(s)) d s+\gamma \delta_{f} t .
$$

In fact, from $\left(H_{1}\right)-\left(H_{3}\right)$ and Lemma 7, for each $r \epsilon$ $(0,1), s a(s) f(u(s)) \in L^{1}[0, r]$ and $(1-s) a(s) f(u(s)) \quad \in$ $L^{1}[r, 1]$. Combining the continuity of $s a(s) f(u(s))$ and $(1-$ s) $a(s) f(u(s))$, we have

$$
\begin{gathered}
\int_{0}^{t} s a(s) f(u(s)) d s \in C^{1}(0, r], \\
\int_{t}^{1}(1-s) a(s) f(u(s)) d s \in C^{1}[r, 1) .
\end{gathered}
$$

Thus $v(t) \in C^{1}(0,1)$. Moreover

$$
\begin{aligned}
v^{\prime}(t)= & -\int_{0}^{t} s a(s) f(u(s)) d s \\
& +\int_{t}^{1}(1-s) a(s) f(u(s)) d s+\gamma \delta_{f} .
\end{aligned}
$$

Similarity, $v^{\prime}(t) \in C^{1}(0,1)$. From (15), we get $v^{\prime \prime}(t)=$ $-a(t) f(u(t)), t \in(0,1)$.

By Lemma 7, we have from (13) that

$$
\begin{aligned}
v(0)= & \lim _{t \rightarrow 0^{+}} v(t) \\
= & \lim _{t \rightarrow 0^{+}}(1-t) \int_{0}^{t} s a(s) f(u(s)) d s \\
& +\lim _{t \rightarrow 0^{+}} t \int_{t}^{1}(1-s) a(s) f(u(s)) d s \\
& +\lim _{t \rightarrow 0^{+}} t \gamma \delta_{f}=0 .
\end{aligned}
$$

Again applying (13), we have

$$
v(1)=\lim _{t \rightarrow 1^{-}} v(t)=\gamma \delta_{f} .
$$

This together with (13) implies that $v(1)=\sum_{i=1}^{m-2} \alpha_{i} v\left(\xi_{i}\right)$. The claim is proved.

By Lemma 7, we obtain from (11), (12), and (13) that

$$
\begin{aligned}
u(t)= & \int_{0}^{t} v(\tau) d \tau \\
= & \int_{0}^{t} \int_{0}^{\tau}(1-\tau) s a(s) f(u(s)) d s d \tau \\
& +\int_{0}^{t} \int_{\tau}^{1}(1-s) \tau a(s) f(u(s)) d s d \tau+\frac{1}{2} \gamma \delta_{f} t^{2} \\
= & \frac{1}{2} \int_{0}^{t}\left(2 t-t^{2}-s\right) s a(s) f(u(s)) d s \\
& +\frac{1}{2} \int_{t}^{1}(1-s) t^{2} a(s) f(u(s)) d s+\frac{1}{2} \gamma \delta_{f} t^{2} .
\end{aligned}
$$

It is easy to see that $u \in C^{1}[0,1] \cap C^{3}(0,1)$, and moreover, $u$ is a nonnegative solution of the BVP (6).

The proof is complete.

Lemma 9 (see [14, Lemmas 2.2 and 2.3]). For any $(t, s) \in$ $[0,1] \times[0,1]$, one has

(i) $q(t) G(1, s) \leq G(t, s) \leq G(1, s)=(1 / 2)(1-s)$ s, where $q(t)=t^{2}$,

(ii) $(\partial / \partial t) G(t, s)=G_{1}(t, s)$, and $0 \leq G_{1}(t, s) \leq G_{1}(s, s)=$ $(1-s) s$.

Lemma 10. Suppose that $\left(H_{1}\right)-\left(H_{3}\right)$ hold; then the unique nonnegative solution $u$ of (6) satisfies

$$
\min _{t \in[\theta, 1]} u(t) \geq \theta^{2}\|u\| .
$$

The proof is similar to Lemma 2.4 in [14].

Lemma 11. Suppose that $\left(H_{1}\right)-\left(H_{3}\right)$ hold. Let $j \in$ $\{1,2, \ldots, m-2\}$ and $r \in(-\infty, 0)$. Then $B V P$

$$
w^{\prime \prime}(t)=0, \quad 0<\tau<t<1,
$$

$$
w(\tau)=0, \quad w(1)-\sum_{i=j}^{m-2} \alpha_{i} w\left(\xi_{i}\right)=r
$$

has a unique solution $w$ satisfying $w(t) \leq 0$ on $[\tau, 1]$.

Proof. From (20) and (21), we obtain $w(t)=(t-\tau) w^{\prime}(\tau)$. Again applying (21), we have

$$
\begin{gathered}
(1-\tau) w^{\prime}(\tau)-\sum_{i=j}^{m-2} \alpha_{i}\left(\xi_{i}-\tau\right) w^{\prime}(\tau)=r, \\
w^{\prime}(\tau)\left(1-\tau-\sum_{i=j}^{m-2} \alpha_{i}\left(\xi_{i}-\tau\right)\right)=r .
\end{gathered}
$$

Now set $d:=1-\tau-\sum_{i=j}^{m-2} \alpha_{i}\left(\xi_{i}-\tau\right)$; then we have that

$$
\begin{aligned}
d & >1-\tau-\sum_{i=j}^{m-2} \alpha_{i}\left(\xi_{i}-\tau \xi_{i}\right) \\
& =(1-\tau)\left(1-\sum_{i=j}^{m-2} \alpha_{i} \xi_{i}\right)>0 .
\end{aligned}
$$

This together with the fact that $r \in(-\infty, 0)$ implies that $w^{\prime}(\tau)<0$. Thus $w(t) \leq 0$ on $[\tau, 1]$. The proof is complete.

\section{Proof of the Main Results}

In this section, we will prove our main results.

Proof of Theorem 1. We divide the proof into three steps.

Step 1. We first prove the existence of positive solutions to (1), $\left(2_{\lambda}\right)$ for sufficiently small $\lambda: \lambda>0$. 
Let $h$ be the unique solution of

$$
\begin{gathered}
u^{\prime \prime \prime}(t)=0, \quad 0<t<1, \\
u(0)=u^{\prime}(0)=0, \quad u^{\prime}(1)-\sum_{i=1}^{m-2} \alpha_{i} u^{\prime}\left(\xi_{i}\right)=1 .
\end{gathered}
$$

Then $h(t)=(1 / 2) \gamma t^{2}$. Let $v=u-\lambda h$; then $u$ is a positive solution of BVP (1), $\left(2_{\lambda}\right)$ if and only if $v=u-\lambda h$ is a nonnegative solution of BVP

$$
\begin{aligned}
& v^{\prime \prime \prime}(t)+a(t) f(v(t)+\lambda h(t))=0, \quad 0<t<1, \\
& v(0)=v^{\prime}(0)=0, \quad v^{\prime}(1)-\sum_{i=1}^{m-2} \alpha_{i} v^{\prime}\left(\xi_{i}\right)=0 .
\end{aligned}
$$

Let $\tilde{f}(x)=\sup _{0 \leq s \leq x} f(s)$. Since $\lim _{u \rightarrow 0^{+}} \sup (\tilde{f}(u) / u)<$ $\Lambda_{1}$; then there exists a positive number $\lambda_{1}$ such that

$$
\tilde{f}\left(\lambda_{1}+\lambda_{1}\|h\|\right) \leq \Lambda_{1}\left(\lambda_{1}+\lambda_{1}\|h\|\right)=2 \lambda_{1} L .
$$

Define a closed convex subset in $C^{+}[0,1]$ by

$$
D=\left\{v \in C^{+}[0,1] \mid v(t) \leq \lambda_{1}, t \in[0,1]\right\}
$$

and an operator $T: D \rightarrow C^{+}[0,1]$ by

$$
\begin{aligned}
T v(t):=\int_{0}^{1} G(t, s) a(s) f( & (s) \\
& +\lambda h(s)) d s+\frac{1}{2} \gamma \delta_{f} t^{2}
\end{aligned}
$$

where $\delta_{f}=\sum_{i=1}^{m-2} \alpha_{i} \int_{0}^{1} G_{1}\left(\xi_{i}, s\right) a(s) f(v(s)+\lambda h(s)) d s$. Modeling the proof of Lemma 2.3 in [10], we can show that $T$ is a completely continuous operator. From Lemma 8, we know that $v$ is a nonnegative solution of (25) if and only if $v$ is a fixed point of $T$.

Suppose that $\lambda<\lambda_{1}$; we claim that $T: D \rightarrow D$.

In fact, from Lemma 9 and (28), we have

$$
\begin{aligned}
& 0 \leq T v(t) \\
& \leq \widetilde{f}\left(\lambda_{1}+\lambda_{1}\|h\|\right)[ \frac{1}{2} \int_{0}^{1}(1-s) s a(s) d s \\
&\left.+\frac{1}{2} \gamma \sum_{i=1}^{m-2} \alpha_{i} \int_{0}^{1}(1-s) s a(s) d s\right] \\
&=\frac{1}{2} \widetilde{f}\left(\lambda_{1}+\lambda_{1}\|h\|\right) L^{-1} \leq \lambda_{1} .
\end{aligned}
$$

The claim is proved. Using the Schauder fixed point theorem, we conclude that $T$ has a fixed point $v$ in $D$, and then $u=v+\lambda h$ is a positive solution of $(1),(2 \lambda)$.

Step 2. We verify that BVP of (1), $\left(2_{\lambda}\right)$ has no positive solutions for $\lambda$ large enough.

Suppose to the contrary that BVP of $(1),(2 \lambda)$ has at least one positive solution for any $\lambda>0$. Then there exist $0<\lambda_{1}<\lambda_{2}<\cdots<\lambda_{n}<\cdots$, with $\lim _{n \rightarrow \infty} \lambda_{n}=\infty$, such that for any positive integer $n$, BVP of $(1),\left(2_{\lambda}\right)$ has a positive solution $u_{n}$. Thus $v_{n}=u_{n}-\lambda_{n} h$ is a nonnegative solution to (25). On the one hand, we have

$$
\left\|v_{n}+\lambda_{n} h\right\| \geq \lambda_{n}\|h\|=\frac{1}{2} \gamma \lambda_{n} \longrightarrow \infty, \quad(n \longrightarrow \infty) .
$$

On the other hand, since $\lim _{u \rightarrow+\infty} \inf (f(u) / u)>\Lambda_{2}$, there exists $r_{0}>0$ such that $f(u) \geq \Lambda_{2} u$, for any $u \in\left[r_{0}, \infty\right)$. Let $n$ be large enough that $\theta^{2}\left\|v_{n}+\lambda_{n} h\right\| \geq r_{0}$. By Lemma 10, we have

$$
\begin{gathered}
\inf _{t \in[\theta, 1]} v_{n}(t) \geq \theta^{2}\left\|v_{n}\right\|, \\
\inf _{t \in[\theta, 1]} h(t)=\frac{1}{2} \gamma \theta^{2}=\theta^{2}\|h\| .
\end{gathered}
$$

This implies that

$$
\inf _{t \in[\theta, 1]}\left(v_{n}(t)+\lambda_{n} h(t)\right) \geq \theta^{2}\left(\left\|v_{n}\right\|+\lambda_{n}\|h\|\right) \geq \theta^{2}\left\|v_{n}+\lambda_{n} h\right\| .
$$

Thus

$$
\begin{aligned}
\| v_{n} & +\lambda_{n} h \| \\
\geq & \left\|v_{n}\right\|=\int_{0}^{1} G(1, s) a(s) f\left(v_{n}(s)+\lambda_{n} h(s)\right) d s \\
& \quad+\frac{1}{2} \gamma \sum_{i=1}^{m-2} \alpha_{i} \int_{0}^{1} G_{1}\left(\xi_{i}, s\right) a(s) f\left(v_{n}(s)+\lambda_{n} h(s)\right) d s \\
\geq & \int_{\theta}^{1} G(1, s) a(s) f\left(v_{n}(s)+\lambda_{n} h(s)\right) d s \\
\geq & \frac{1}{2} \Lambda_{2}\left(v_{n}(s)+\lambda_{n} h(s)\right) \int_{\theta}^{1}(1-s) s a(s) d s \\
= & 2\left\|v_{n}+\lambda_{n} h\right\|,
\end{aligned}
$$

which is a contradiction.

Step 3. Let $B=\left\{\lambda \mid\right.$ BVP of (1), $\left(2_{\lambda}\right)$ has at least one positive solution $\}$ and $\lambda^{*}=\sup B$; then $0<\lambda^{*}<\infty$. We show that (1), $\left(2_{\lambda}\right)$ have positive solution for any $\lambda \in\left(0, \lambda^{*}\right)$. From the definition of $\lambda^{*}$, we know that, for any $\lambda \in\left(0, \lambda^{*}\right)$, there exists $\tilde{\lambda}>\lambda$ such that $(1),\left(2_{\lambda}\right)$ have positive solution $u_{\tilde{\lambda}}$.

Now we consider the following third-order $m$-point boundary value problem:

$$
\begin{gathered}
u^{\prime \prime \prime}(t)+a(t)(\widetilde{F} u)(t)=0, \quad 0<t<1, \\
u(0)=u^{\prime}(0)=0, \quad u^{\prime}(1)-\sum_{i=1}^{m-2} \alpha_{i} u^{\prime}\left(\xi_{i}\right)=\lambda,
\end{gathered}
$$

where

$$
(\widetilde{F} u)(t)= \begin{cases}f\left(u_{\tilde{\lambda}}(t)\right), & \text { if } u(t)>u_{\tilde{\lambda}}(t), \\ f(u(t)), & \text { if } 0 \leq u(t) \leq u_{\tilde{\lambda}}(t), \\ f(0), & \text { if } u(t)<0 .\end{cases}
$$


Since $\widetilde{F}$ is bounded, by Schauder fixed point theorem, the problem (34) has a solution $u_{\lambda}$.

By Lemma $8, u_{\lambda}$ satisfies

$$
\begin{aligned}
u_{\lambda}(t)= & \int_{0}^{1} G(t, s) a(s)(\widetilde{F} u)(s) d s \\
& +\frac{1}{2} \gamma t^{2} \sum_{i=1}^{m-2} \alpha_{i} \int_{0}^{1} G_{1}\left(\xi_{i}, s\right) a(s) \times(\widetilde{F} u)(s) d s \\
& +\frac{1}{2} \gamma \lambda t^{2},
\end{aligned}
$$

thus $u_{\lambda} \geq 0$.

Let $I=\left\{t \in(0,1] \mid u_{\lambda}(t)>u_{\tilde{\lambda}}(t)\right\}$ and $\Omega=\{t \in(0,1] \mid$ $w(t)>0\}$, where $w(t)=u_{\lambda}^{\prime}(t)-u_{\tilde{\lambda}}^{\prime}(t), t \in[0,1]$. We will show that $I=\emptyset$. Noticing that, if $u_{\lambda}^{\prime}(t)>u_{\tilde{\lambda}}^{\prime}(t)$ holds for any $t \in(0,1)$, combining with $u_{\lambda}^{\prime}(0)=u_{\tilde{\lambda}}^{\prime}(0)$, we get $u_{\lambda}(t)>u_{\tilde{\lambda}}(t)$. Thus we prove that $\Omega=\emptyset$; then we have $I=\emptyset$.

Suppose to the contrary that $\Omega \neq \emptyset$.

If $u_{\lambda}^{\prime}(1)<u_{\tilde{\lambda}}^{\prime}(1)$, then, from $\Omega \neq \emptyset$, and the continuity of $w(t)$, there exists $(a, b) \subset \Omega$ such that $w^{\prime}(a)=w(b)=0$. Moreover, $w^{\prime \prime}(t)=0$ in $(a, b)$. Thus $w(t) \equiv 0$ in $(a, b)$. This contradicts with the fact that $w(t)>0$ in $(a, b)$.

If $u_{\lambda}^{\prime}(1)>u_{\tilde{\lambda}}^{\prime}(1)$, we claim that there exists $j \in$ $\{1,2, \ldots, m-2\}$ such that $w\left(\xi_{j}\right)>0$.

In fact, from the fact that $w(1)-\sum_{i=1}^{m-2} \alpha_{i} w\left(\xi_{i}\right)=\lambda-\widetilde{\lambda}<0$ and $w(1)>0$, we have that $\sum_{i=1}^{m-2} \alpha_{i} w\left(\xi_{i}\right)>0$. Thus, there exists $j \in\{1,2, \ldots, m-2\}$ such that $w\left(\xi_{j}\right)>0$.

Let $j_{0}=\min \left\{j \mid j \in\{1,2, \ldots, m-2\}\right.$ such that $\left.w\left(\xi_{j}\right)>0\right\}$; then, we only need to deal with the following four cases.

Case 1. $w(t)>0$ in $(0,1)$. In this case, we have

$$
\begin{gathered}
w^{\prime \prime}(t)=0, \quad 0<t<1, \\
w(0)=0, \quad w(1)-\sum_{i=1}^{m-2} \alpha_{i} w\left(\xi_{i}\right)=\lambda-\tilde{\lambda}<0 .
\end{gathered}
$$

We easily verify that

$$
w(t)=(\lambda-\tilde{\lambda}) \gamma t<0, \quad \text { in }(0,1)
$$

This contradicts with the fact that $\Omega \neq \emptyset$.

Case 2. There exists $\tau \in(0,1)$ such that $w(\tau)=0$ and $w(t)>0$ in $(0, \tau)$. In this case, we have

$$
\begin{gathered}
w^{\prime \prime}(t)=0, \quad 0<t<\tau, \\
w(0)=0, \quad w(\tau)=0 .
\end{gathered}
$$

We easily obtain $w(t) \equiv 0$ in $[0, \tau]$, a contradiction again.

Case 3. There exists $\tau \in(0,1)$ such that $w(\tau)=0$ and $w(t)>0$ in $(\tau, 1]$. In this case, if $j_{0}>1$, then, for any $i \in\left\{1,2, \ldots, j_{0}-\right.$ $1\}$, we have $w\left(\xi_{i}\right)<0$. Thus

$$
\begin{aligned}
r: & =w(1)-\sum_{i=j_{0}}^{m-2} \alpha_{i} w\left(\xi_{i}\right) \\
& =w(1)-\sum_{i=1}^{m-2} \alpha_{i} w\left(\xi_{i}\right)+\sum_{i=1}^{j_{0}-1} \alpha_{i} w\left(\xi_{i}\right) \\
& =\lambda-\tilde{\lambda}+\sum_{i=1}^{j_{0}-1} \alpha_{i} w\left(\xi_{i}\right) \\
& \leq \lambda-\tilde{\lambda} \\
& <0 .
\end{aligned}
$$

If $j_{0}=1$, then $r:=w(1)-\sum_{i=j_{0}}^{m-2} \alpha_{i} w\left(\xi_{i}\right)=\lambda-\tilde{\lambda}<0$, and $w(t)$ satisfies

$$
\begin{gathered}
w^{\prime \prime}(t)=0, \quad \tau<t<1, \\
w(\tau)=0, \quad w(1)-\sum_{i=j_{0}}^{m-2} \alpha_{i} w\left(\xi_{i}\right)=r<0 .
\end{gathered}
$$

By Lemma 11, we also have $w(t) \leq 0$ in $[\tau, 1]$, a contradiction again.

Case 4. There exists $[a, b] \subset(0,1)$ such that $w(a)=w(b)=0$ and $w(t)>0$ in $(a, b)$. The same as Case 2 , we can lead to a contradiction.

Summarizing the previous discussion, we assert that $\Omega=$ $\emptyset$; thus $I=\emptyset$. Up to now, the problem $(1),\left(2_{\lambda}\right)$ has a solution $u_{\lambda}$.

Proof of Theorem 2. Since $\lim _{u \rightarrow 0^{+}} \inf (f(u) / u)>0$, there exists $\mu>0$, and $r_{1}>0$ such that $f(u) \geq \mu u, u \in\left[0, r_{1}\right]$. Next we consider two cases: $f$ is bounded or $f$ is unbounded.

Case 1. Suppose that $f$ is bounded, that is, $f(u) \leq M$, for all $u \in[0, \infty)$. By Schauder fixed point theorem the problem of $(1),\left(2_{\lambda}\right)$ has a positive solution.

Case 2. If $f$ is unbounded. Since $\lim _{u \rightarrow+\infty} \sup (f(u) / u)<L$, there exists a positive number $r_{2}$ such that $f(u) \leq L u$, for $u \in\left[r_{2}, \infty\right)$. Since $f$ is unbounded, for any $\lambda \in(0, \infty)$, we are able to choose

$$
R_{\lambda} \geq \max \left\{2 r_{1}, r_{2}, \lambda\|h\|\right\}
$$

such that

$$
f(u) \leq f\left(2 R_{\lambda}\right), \quad \text { for } u \in\left[0,2 R_{\lambda}\right] .
$$

Defining a closed convex subset in $C^{+}[0,1]$ by

$$
D_{1}=\left\{v \in C^{+}[0,1] \mid v(t) \leq R_{\lambda}, t \in[0,1]\right\} \text {. }
$$


For each $v \in D_{1}$, we have $0 \leq v+\lambda h \leq 2 R_{\lambda}$. By (28) and Lemma 9, we obtain that

$$
\begin{aligned}
T v(t) \leq \frac{1}{2} f\left(2 R_{\lambda}\right) & \left(\int_{0}^{1}(1-s) s a(s) d s\right. \\
& \left.+\gamma \sum_{i=1}^{m-2} \alpha_{i} \int_{0}^{1}(1-s) s a(s) d s\right) \\
\leq & \frac{1}{2} L \cdot 2 R_{\lambda} L^{-1}=R_{\lambda} .
\end{aligned}
$$

That is, $T v(t) \in D_{1}$. By using the Schauder fixed point theorem, we assert that $T$ has a fixed point $v \in D_{1}$, and then $u=v+\lambda h$ is a positive solution of BVP of $(1),(2 \lambda)$.

\section{Example}

Consider the boundary value problem

$$
\begin{array}{r}
u^{\prime \prime \prime}(t)+\frac{1}{t(1-t)} \cdot \frac{u^{2}(t)+u(t)}{u(t)+176}(7+\cos u(t))=0, \\
0<t<1, \\
u(0)=u^{\prime}(0)=0, \quad u^{\prime}(1)-\sum_{i=1}^{4} i u^{\prime}\left(\frac{1}{8 i}\right)=\lambda,
\end{array}
$$

where $\alpha_{i}=i, \xi_{i}=(1 / 8 i)(i=1,2,3,4), a(t)=1 / t(1-t), t \in$ $(0,1)$, and $f(u)=\left(\left(u^{2}+u\right) /(u+176)\right)(7+\cos u), u \in[0,+\infty)$. Obviously $\left(H_{1}\right)$, and $\left(H_{2}\right)$, and $\left(H_{3}\right)$ hold. By calculating, we have $\gamma=2,\|h\|=1, L=1 / 21, \Lambda_{1}=1 / 21$. Let $\theta=1 / 4$, then $\Lambda_{2}=16 / 3$. We easily verify that

$$
\lim _{u \rightarrow 0^{+}} \sup \frac{f(u)}{u}=\frac{1}{22}<\Lambda_{1}, \quad \lim _{u \rightarrow+\infty} \inf \frac{f(u)}{u}=6>\Lambda_{2},
$$

that is, $\left(H_{4}\right)$ is satisfied. Therefore, Theorem 1 now guarantees that there exists a positive number $\lambda^{*}$ such that BVP of (46), $\left(47_{\lambda}\right)$ has at least one positive solution for $\lambda \in\left(0, \lambda^{*}\right)$ and none for $\lambda \in\left(\lambda^{*}, \infty\right)$.

But we cannot apply Theorem B [14, Theorem 3.2]. In fact, $f$ does not satisfy monotonicity condition. Moreover, condition $\left(H_{4}^{*}\right)$ of Theorem B does not hold.

\section{Acknowledgments}

The authors are grateful to the anonymous referee for his or her constructive comments and suggestions which led to improvement of the original paper. X. Han is supported by the NNSF of China (no.11101335).

\section{References}

[1] R. P. Agarwal, "On boundary value problems for $y^{\prime \prime \prime}=$ $f\left(x, y, y^{\prime}, y^{\prime \prime}\right)$, , Bulletin of the Institute of Mathematics, vol. 12, no. 2, pp. 153-157, 1984.
[2] L. K. Jackson, "Boundary value problems for ordinary differential equations," in Studies in Differential Equations, J. Hale, Ed., vol. 14, pp. 93-127, The Mathematical Association of America, Washington, DC, USA, 1977.

[3] D. R. K. S. Rao, K. N. Murthy, and A. S. Rao, "Threepoint boundary value problems associated with third order differential equations," Nonlinear Analysis, vol. 5, no. 6, pp. 669673, 1981.

[4] C. P. Gupta, "Solvability of a three-point nonlinear boundary value problem for a second order ordinary differential equation," Journal of Mathematical Analysis and Applications, vol. 168, no. 2, pp. 540-551, 1992.

[5] D. Anderson, "Multiple positive solutions for a three-point boundary value problem," Mathematical and Computer Modelling, vol. 27, no. 6, pp. 49-57, 1998.

[6] R. Ma, "Multiplicity results for a third order boundary value problem at resonance," Nonlinear Analysis. Theory, Methods \& Applications, vol. 32, no. 4, pp. 493-499, 1998.

[7] A. Cabada and S. Heikkilä, "Extremality and comparison results for discontinuous third order functional initial-boundary value problems," Journal of Mathematical Analysis and Applications, vol. 255, no. 1, pp. 195-212, 2001.

[8] A. Cabada, "The method of lower and upper solutions for second, third, fourth, and higher order boundary value problems," Journal of Mathematical Analysis and Applications, vol. 185, no. 2, pp. 302-320, 1994.

[9] L.-J. Guo, J.-P. Sun, and Y.-H. Zhao, "Existence of positive solutions for nonlinear third-order three-point boundary value problems," Nonlinear Analysis. Theory, Methods \& Applications, vol. 68, no. 10, pp. 3151-3158, 2008.

[10] S. Li, "Positive solutions of nonlinear singular third-order two-point boundary value problem," Journal of Mathematical Analysis and Applications, vol. 323, no. 1, pp. 413-425, 2006.

[11] M. El-Shahed, "Positive solutions for nonlinear singular third order boundary value problem," Communications in Nonlinear Science and Numerical Simulation, vol. 14, no. 2, pp. 424-429, 2009.

[12] Q. Yao, "Positive solutions of singular third-order three-point boundary value problems," Journal of Mathematical Analysis and Applications, vol. 354, no. 1, pp. 207-212, 2009.

[13] R. Ma, "Positive solutions for second-order three-point boundary value problems," Applied Mathematics Letters, vol. 14, no. 1, pp. 1-5, 2001.

[14] Y. Sun, "Positive solutions for third-order three-point nonhomogeneous boundary value problems," Applied Mathematics Letters, vol. 22, no. 1, pp. 45-51, 2009.

[15] H. Asakawa, "Nonresonant singular two-point boundary value problems," Nonlinear Analysis. Theory, Methods \& Applications, vol. 44, no. 6, pp. 791-809, 2001. 


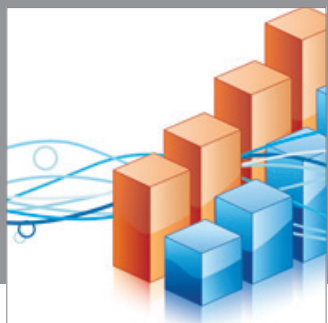

Advances in

Operations Research

mansans

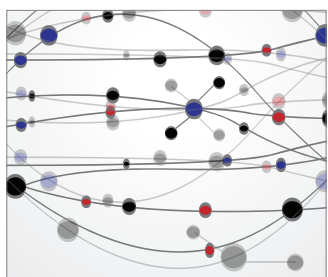

The Scientific World Journal
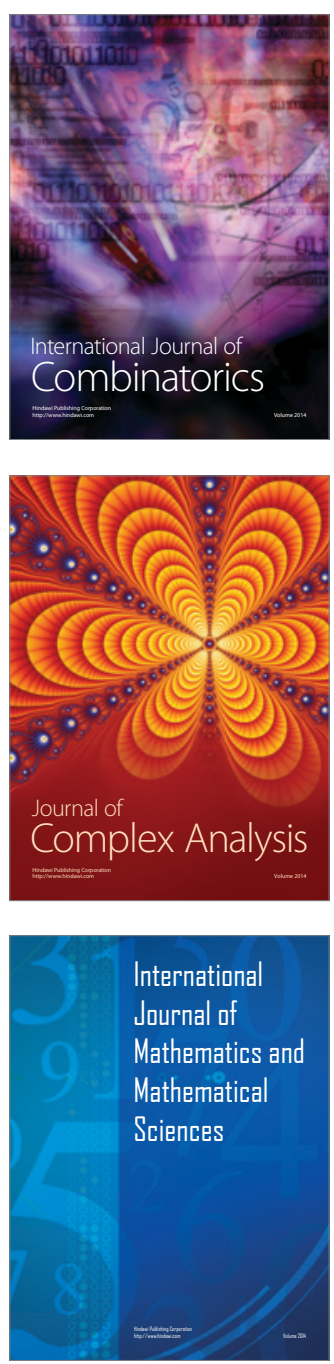
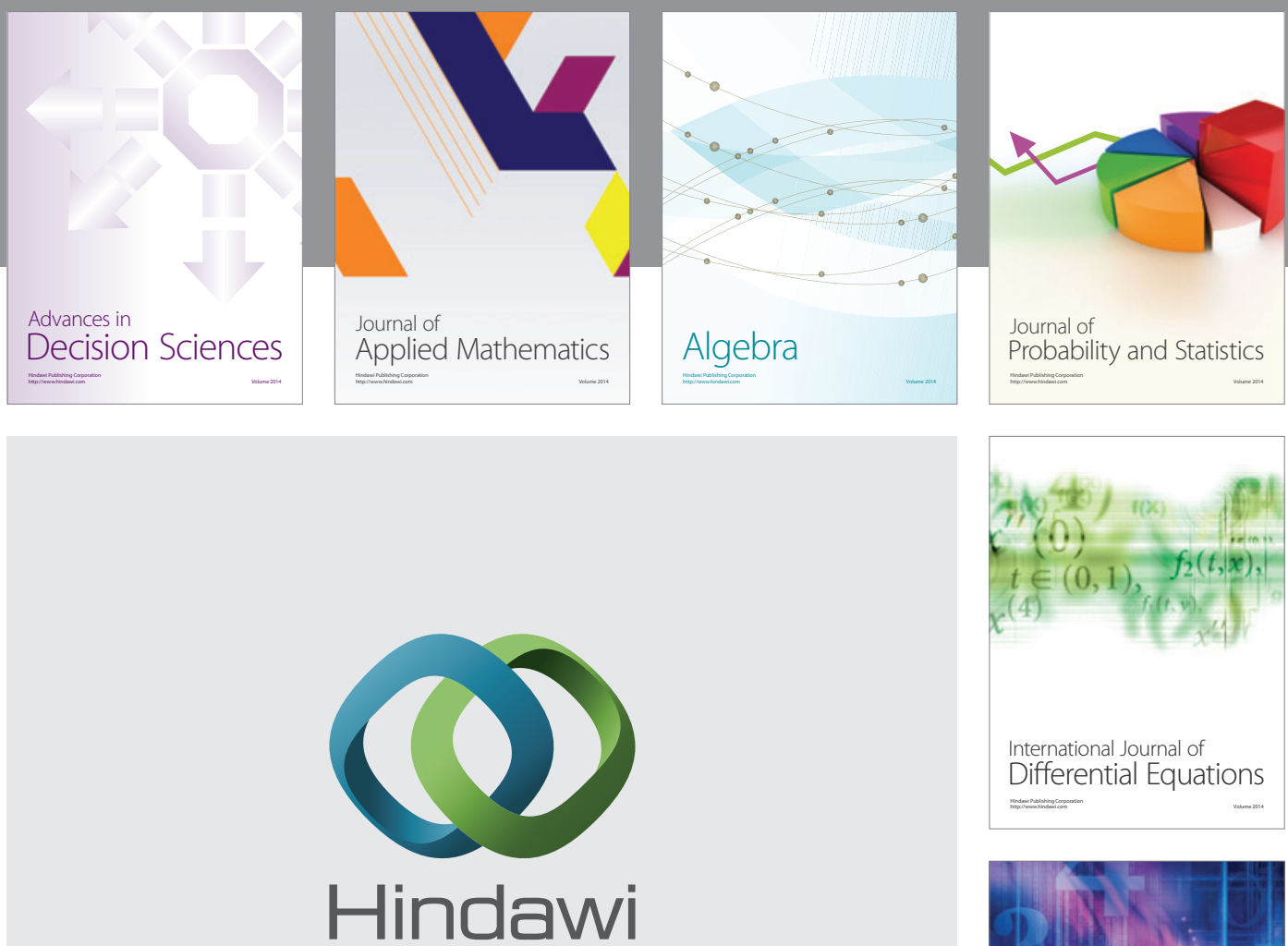

Submit your manuscripts at http://www.hindawi.com
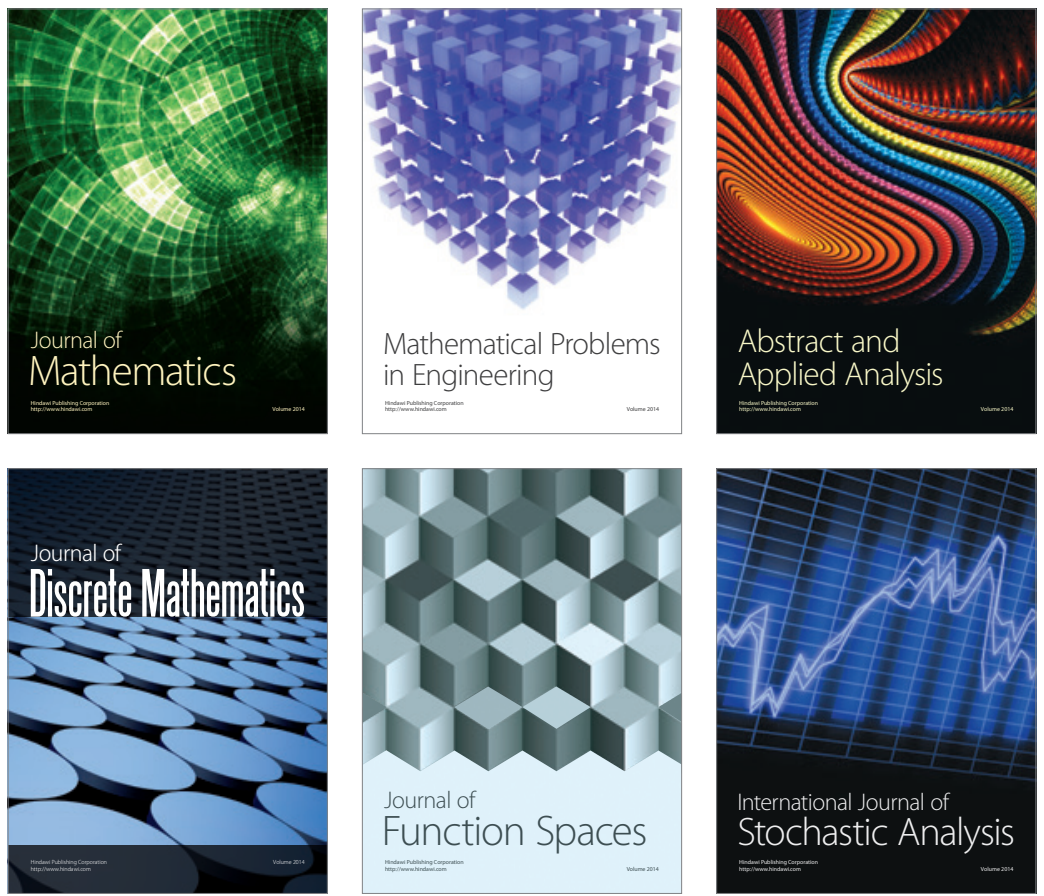

Journal of

Function Spaces

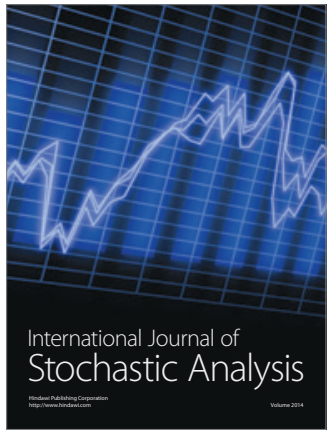

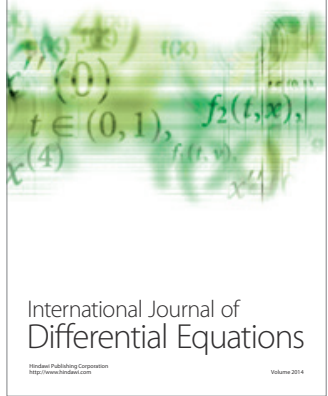
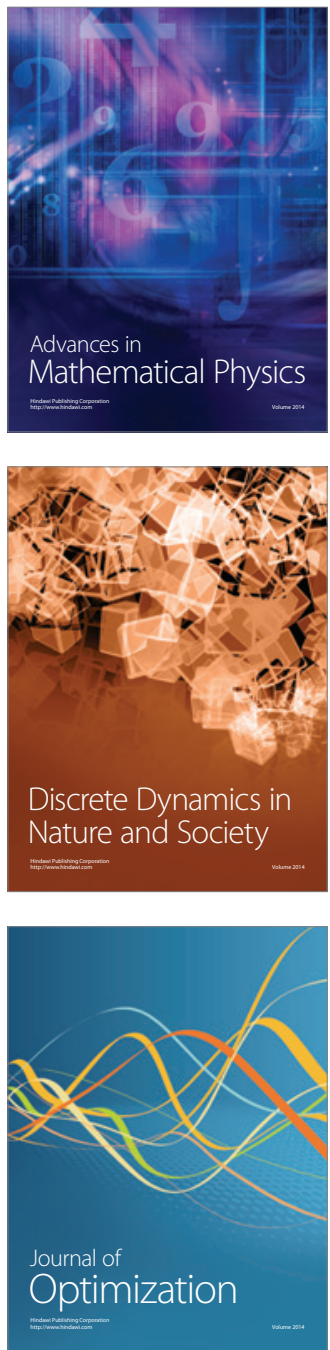